PROCEEDINGS OF THE

AMERICAN MATHEMATICAL SOCIETY

Volume 132, Number 12, Pages 3615-3625

S 0002-9939(04)07477-5

Article electronically published on July 20, 2004

\title{
ARE GENERALIZED LORENTZ "SPACES" REALLY SPACES?
}

\author{
MICHAEL ĆWIKEL, ANNA KAMIŃSKA, LECH MALIGRANDA, AND LUBOŠ PICK \\ (Communicated by N. Tomczak-Jaegermann)
}

\begin{abstract}
We show that the Lorentz space $\Lambda^{p}(w)$ need not be a linear set for certain "non-classical" weights $w$. We establish necessary and sufficient conditions on $p$ and $w$ for this situation to occur.
\end{abstract}

\section{Introduction And Statement of Main RESUlts}

Let $f$ be a real-valued measurable function on $\mathbb{R}$. We define the distribution function of $f$ by $f_{*}(\lambda)=|\{x \in \mathbb{R}:|f(x)|>\lambda\}|$ for each $\lambda>0$, (where $|\cdot|$ denotes Lebesgue measure). The non-increasing rearrangement of $f$ is defined by

$$
f^{*}(t)=\inf \left\{s>0: f_{*}(s) \leq t\right\}, \quad t \in[0, \infty) .
$$

We further denote

$$
f^{* *}(t)=\frac{1}{t} \int_{0}^{t} f^{*}(s) d s, \quad t \in(0, \infty)
$$

When $w$ is a non-negative measurable function on $(0, \infty)$ that is not identically zero, we say that $w$ is a weight. Note that our definition here (which is consistent with the usage in [16], and in essentially all subsequent papers cited below) differs from another frequently used definition in the context of many kinds of function spaces, which requires $w$ to be strictly positive.

Definition 1.1. Let $p \in(0, \infty)$, let $w$ be a weight, and let $W(t)=\int_{0}^{t} w(s) d s$. Suppose that $W(t)<\infty$ for all $t>0$. We define four types of function spaces on

Received by the editors January 21, 2003 and, in revised form, July 16, 2003.

2000 Mathematics Subject Classification. Primary 46E30, 46B42.

Key words and phrases. Lorentz spaces, Marcinkiewicz spaces, Lorentz-Orlicz spaces, weights, rearrangement.

The first named author was supported by the Dent Charitable Trust-Non-Military Research Fund and by the Fund for Promotion of Research at the Technion. The second named author was supported by project no. SMK-2136 of the Kempe Foundation in Sweden. The third named author was supported by the Swedish Natural Science Research Council (NFR)-grant M5105-20005228/2000. The fourth named author was supported by grant no. 201/01/0333 of the Grant Agency of the Czech Republic and by grant no. MSM 113200007 of the Czech Ministry of Education. 
$\mathbb{R}$ by

$$
\begin{cases}\Lambda^{p}(w) & =\left\{f \text { measurable }:\|f\|_{\Lambda^{p}(w)}:=\left(\int_{0}^{\infty}\left(f^{*}(t)\right)^{p} w(t) d t\right)^{1 / p}<\infty\right\} ; \\ \Lambda^{p, \infty}(w) & =\left\{f \text { measurable }:\|f\|_{\Lambda^{p, \infty}(w)}:=\sup _{0<t<\infty} f^{*}(t) W^{1 / p}(t)<\infty\right\} \\ \Gamma^{p}(w) & =\left\{f \text { measurable }:\|f\|_{\Gamma^{p}(w)}:=\left(\int_{0}^{\infty}\left(f^{* *}(t)\right)^{p} w(t) d t\right)^{1 / p}<\infty\right\} \\ \Gamma^{p, \infty}(w) & =\left\{f \text { measurable }:\|f\|_{\Gamma^{p, \infty}(w)}:=\sup _{0<t<\infty} f^{* *}(t) W^{1 / p}(t)<\infty\right\} .\end{cases}
$$

The spaces $\Lambda^{p}(w)$ were introduced by Lorentz in 1951 in [16. Spaces whose norms involve $f^{* *}$ appeared explicitly for the first time in Calderón's paper [5]. In 22] Sawyer considered $\Gamma^{p}(w)$ for arbitrary weights, in connection with the study of certain duality problems concerning the spaces $\Lambda^{p}(w)$.

The spaces $\Lambda^{p, \infty}(w)$ and $\Gamma^{p, \infty}(w)$ are called Marcinkiewicz spaces or weak Lorentz spaces (see [15], [11]; see also [8], 9], [10, 6] and 7]).

Lorentz [16] proved that, for $p \geq 1,\|f\|_{\Lambda^{p}(w)}$ is a norm if and only if $w$ is nonincreasing. The class of weights for which $\|f\|_{\Lambda^{p}(w)}$ is merely equivalent to a Banach norm is however considerably larger. In fact it consists of all those weights $w$ which, for some $C$ and all $t>0$, satisfy

$$
t^{p} \int_{t}^{\infty} x^{-p} w(x) d x \leq C \int_{0}^{t} w(x) d x \quad \text { when } p \in(1, \infty)
$$

(22, Theorem 4], see also [1]), or

$$
\frac{1}{t} \int_{0}^{t} w(x) d x \leq \frac{C}{s} \int_{0}^{s} w(x) d x \quad \text { for } 0<s \leq t \quad \text { when } p=1
$$

([6, Theorem 2.3]). It is also known that, for $p \in[1, \infty)$, the space $\Lambda^{p}(w)$ is equivalent to a Banach space if and only if the Hardy-Littlewood maximal operator acts boundedly from $\Lambda^{p}(w)$ to $\Lambda^{p, \infty}(w)$ ([9] Theorem 3.3], [10, Theorem 3.9], [6]). Furthermore, for $p>1$, this is equivalent to $\Lambda^{p}(w)=\Gamma^{p}(w)$ ([22]).

In [11, Th. 1.1] (see also [8, Corollary 2.2], [13, p. 6]) it was observed that the functional $\|f\|_{\Lambda^{p}(w)}, 0<p \leq \infty$, does not have to be a quasinorm. It was shown that it is a quasinorm if and only if the function $W$ satisfies the $\Delta_{2}$-condition, i.e.,

$$
W(2 t) \leq C W(t) \quad \text { for some } C>1 \text { and all } t \in(0, \infty) .
$$

There are a number of natural and important function spaces which are not normable, such as weak $L^{1}$ and $H^{p}$ and $L^{p}$ and $L^{p, q}$ for $p<1$. They exhibit various different behaviours with respect to the existence of non-trivial dual spaces.

In this note we encounter different, perhaps more exotic behaviour in the case of generalized Lorentz spaces. It can happen that the sum of two functions in the "space" is not in the space.

Obviously $f \in \Lambda^{p}(w)$ implies that $\lambda f \in \Lambda^{p}(w)$ for every $\lambda \in \mathbb{R}$. However in general, perhaps surprisingly, $\Lambda^{p}(w)$ is not a linear space. In this note we shall give necessary and sufficient conditions for $\Lambda^{p}(w)$ to be a linear space.

In fact, other function spaces that are not closed with respect to addition sometimes appear quite naturally in analysis. One example going back to the 1930's is the Orlicz class $L^{\varphi}=\left\{f\right.$ measurable $\left.: \int_{\mathbb{R}} \varphi(|f(t)|) d t<\infty\right\}$, which is linear if and only if $\varphi$ satisfies the $\Delta_{2}$-condition (see [19], where this was proved even in the case where $\varphi$ is only a Baire function). The spaces of functions of bounded $\varphi$-variation $B V_{\varphi}=\left\{f:[a, b] \rightarrow \mathbb{R}: \sup _{P} \sum_{k=1}^{n} \varphi\left(\left|f\left(x_{k}\right)-f\left(x_{k-1}\right)\right|\right)<\infty\right\}$, where the supremum is taken over all partitions $P$ of $[a, b]$, are linear spaces if and only 
if $\varphi$ satisfies the $\Delta_{2}$-condition near zero (see [20]; cf. also [21]). The Wiener spaces ([23]) $\left\{f\right.$ measurable : $\left.\lim _{T \rightarrow \infty}\left(\frac{1}{2 T} \int_{-T}^{T}|f(t)|^{p} d t\right)^{1 / p}<\infty\right\}$, sometimes also called the spaces of bounded power signals, are not linear spaces ([18]). Note that if instead of "limit" we take either "limit superior" or "supremum", the corresponding spaces will be linear (they are classical Besicovitch-Marcinkiewicz or Stepanoff-Weyl spaces). The "weak $L^{\infty}$ " space $W=\left\{f\right.$ measurable : $f^{*}$ is finite and $\|f\|_{W}:=$ $\left.\sup _{t>0}\left[f^{* *}(t)-f^{*}(t)\right]<\infty\right\}$ of Bennett, DeVore and Sharpley ([3]; see also [4]) and the space $W_{p}$, determined by the "norm"

$$
\|f\|_{W_{p}}:=\left(\int_{0}^{\infty} \frac{\left(f^{*}\left(\frac{t}{2}\right)-f^{*}(t)\right)^{p}}{t} d t\right)^{\frac{1}{p}}<\infty,
$$

defined recently in [17] in connection with sharp limiting Sobolev embeddings, are also examples of spaces that are not linear. The latter two spaces are of special interest in interpolation theory. The space $L(\infty, q)$ of [2] is yet another example, related to the two preceding ones.

Before formulating and proving our main result it is convenient to present a lemma which will be needed later.

Lemma 1.2. Suppose that $w$ and $v$ are non-negative measurable functions on $(0, \infty)$ and $a$ is a positive number such that $w(t)=0$ for a.e. $t \in(0, a)$ and $w(t)=v(t)$ for all $t \geq a$, and the functions $V(t)=\int_{0}^{t} v(x) d x$ and $W(t)=\int_{0}^{t} w(x) d x$ are finite for all $t>0$.

Let $f: \mathbb{R} \rightarrow \mathbb{R}$ be a measurable function. Then the following are equivalent:

(i) $f \in \Lambda^{p}(w)$.

(ii) $\min \{\lambda,|f|\} \in \Lambda^{p}(v)$ for some positive number $\lambda$.

(iii) $\min \{\lambda,|f|\} \in \Lambda^{p}(v)$ for every positive number $\lambda$.

Proof. Clearly we can assume without loss of generality that $f$ is non-negative.

It follows easily from the definitions of the non-increasing rearrangement and the distribution function that, for each constant $\lambda>0$, the non-increasing rearrangement of the function $\min \{f, \lambda\}$ satisfies

$$
(\min \{f, \lambda\})^{*}(t)=\min \left\{f^{*}(t), \lambda\right\} \text { for each } t>0 .
$$

Now suppose that $f \in \Lambda^{p}(w)$. If $f^{*}(a)>0$ we choose $\lambda=f^{*}(a)$. If $f^{*}(a)=0$ then we can choose $\lambda$ however we wish, for example $\lambda=1$. Note that in this latter case we have $f^{*}(t)=0$ for all $t \in[a, \infty)$. In both cases we have

$$
\begin{aligned}
\int_{0}^{\infty}\left(\min \left\{f^{*}(t), \lambda\right\}\right)^{p} v(t) d t & \leq \int_{0}^{a} \lambda^{p} v(t) d t+\int_{a}^{\infty}\left(f^{*}(t)\right)^{p} w(t) d t \\
& \leq \lambda^{p} V(a)+\|f\|_{\Lambda^{p}(w)}^{p}<\infty .
\end{aligned}
$$

This shows that (i) implies (ii).

Now suppose $\min \left\{f, \lambda_{0}\right\} \in \Lambda^{p}(v)$ for some $\lambda_{0}>0$. Then obviously $\min \{f, \lambda\} \in$ $\Lambda^{p}(v)$ for all $\lambda \in\left(0, \lambda_{0}\right]$. For each $\lambda>\lambda_{0}$, let $A=\left\{t>0: f^{*}(t)>\lambda_{0}\right\}$ and $B=(0, \infty) \backslash A$. Then we have

$$
\begin{aligned}
\|\min \{f, \lambda\}\|_{\Lambda^{p}(v)}^{p} & =\int_{0}^{\infty} \min \left\{f^{*}(t), \lambda\right\}^{p} v(t) d t \\
& \leq \int_{A} \lambda^{p} v(t) d t+\int_{B} \min \left\{f^{*}(t), \lambda_{0}\right\}^{p} v(t) d t .
\end{aligned}
$$


The integral over $B$ is of course finite. The set $A$ must be of the form $(0, \alpha)$ for some $\alpha \in(0, \infty]$. If $\alpha<\infty$, then the integral over $A$ equals $\lambda^{p} V(\alpha)$ and is finite. If $\alpha=\infty$, then $\min \left\{f^{*}(t), \lambda_{0}\right\}=\lambda_{0}$ for all $t>0$, and the assumption that $\min \left\{f, \lambda_{0}\right\} \in \Lambda^{p}(v)$ implies that $\int_{0}^{\infty} v(t) d t<\infty$. Thus in both cases we deduce that $\min \{f, \lambda\} \in \Lambda^{p}(v)$, which shows that (ii) implies (iii).

Finally, suppose that $f$ satisfies condition (iii). If $f^{*}(a)=0$, then obviously $\|f\|_{\Lambda^{p}(w)}=0$. If $f^{*}(a)>0$, then we have that $\min \left\{f, f^{*}(a)\right\} \in \Lambda^{p}(v)$. By (1.5) we have $f^{*}(t)=\left(\min \left\{f, f^{*}(a)\right\}\right)^{*}(t)$ for all $t>a$, and so

$$
\|f\|_{\Lambda^{p}(w)}^{p}=\int_{a}^{\infty}\left(\left(\min \left\{f, f^{*}(a)\right\}\right)^{*}(t)\right)^{p} w(t) d t \leq\left\|\min \left\{f, f^{*}(a)\right\}\right\|_{\Lambda^{p}(v)}^{p}<\infty .
$$

Thus in both cases we deduce that $f \in \Lambda^{p}(w)$, which shows that (iii) $\Longrightarrow$ (i) and completes the proof of the lemma.

Remark 1.3. Let us briefly consider what would happen had we not imposed the condition that $W$ is finite. The four spaces introduced in Definition 1.1 could of course still be defined without this condition. If $W(t)=\infty$ for all $t>0$, then each of these spaces is trivial, i.e., it contains only the zero element. In the remaining case, where

$$
0<t_{0}=\sup \{t>0: W(t)<\infty\}<\infty,
$$

the space $\Gamma^{p, \infty}(w)$ is again trivial, and neither $\Lambda^{p}(w)$ nor $\Lambda^{p, \infty}(w)$ is a linear space. To see this, take $f=\chi_{\left(0, t_{1}\right)}$ and $g(s)=f(-s)$ with $t_{0} / 2<t_{1}<t_{0}$. Then $f$ and $g$ are both in $\Lambda^{p}(w)$ and $\Lambda^{p, \infty}(w)$, but $(f+g)^{*}(s)=f^{*}(s / 2)=\chi_{\left(0,2 t_{1}\right)}(s)$ is not in either of these spaces. Note that here $W$ does not satisfy the $\Delta_{2}$-condition.

We stress that (except during the brief discussion in the preceding remark) we invoke Definition 1.1 throughout this paper and so always assume that $W(t)$ is finite for every $t>0$.

Here now are our main results:

Theorem 1.4. The following are equivalent:

(i) $\Lambda^{p}(w)$ is not a linear space.

(ii) There exists a sequence of positive numbers $t_{n}$, tending either to 0 or to $\infty$, such that $W\left(2 t_{n}\right)>2{ }^{n} W\left(t_{n}\right)$ for all $n \in \mathbb{N}$.

(iii) There exists a sequence of positive numbers $t_{n}$ such that $W\left(2 t_{n}\right)>2^{n} W\left(t_{n}\right)$ $>0$ for all $n \in \mathbb{N}$.

Corollary 1.5. The following are equivalent:

(i) $\Lambda^{p}(w)$ is a linear space.

(ii) $^{\prime}$ There exist positive constants $\alpha, \beta$ and $C$ such that $W(2 t) \leq C W(t)$ for all $t \leq \alpha$ and all $t \geq \beta$.

(iii)' There exists a constant $C^{\prime}$ such that one of the following two conditions hold: Either

$(\text { iii-A })^{\prime}$

$$
W(2 t) \leq C^{\prime} W(t)
$$

for all $t>0$, or

(iii-B) $)^{\prime} W(t)=0$ on some interval $(0, a)$, and (1.6) holds for all $t$ in some interval $(b, \infty)$. 
The conditions for linearity of Marcinkiewicz spaces $\Lambda^{p, \infty}(w)$ are the same as for linearity of $\Lambda^{p}(w)$. Below we formulate only one condition. Notice that (iii) of Theorem 1.4 is the same as (ii) below.

Theorem 1.6. The following are equivalent:

(i) $\Lambda^{p, \infty}(w)$ is not a linear space.

(ii) There exists a sequence of positive numbers $t_{n}$ such that

$$
\frac{W\left(2 t_{n}\right)}{W\left(t_{n}\right)} \rightarrow \infty, \quad n \rightarrow \infty
$$

\section{Proofs}

Joint proof of Theorem 1.4 and Corollary 1.5 . We begin with the easy proof that (ii) implies (iii). If (ii) holds and the sequence $\left\{t_{n}\right\}$ tends to $\infty$, then $W\left(2 t_{1}\right)>0$ and so $W(t)>0$ for all $t \geq 2 t_{1}$. If the original sequence $\left\{t_{n}\right\}$ does not satisfy $W\left(t_{n}\right)>0$ for all $n$, then we simply replace it by a subsequence of numbers in $\left[2 t_{1}, \infty\right)$ and we are done. Alternatively, if (ii) holds and the sequence $\left\{t_{n}\right\}$ tends to 0 , then, since $W\left(2 t_{n}\right)>0$ for all $n \in \mathbb{N}$, we deduce that $W(t)>0$ for all positive $t$ and we obtain condition (iii).

Let us next prove that (iii) implies (i). Given a sequence $\left\{t_{n}\right\}$ satisfying (iii), we let $f=\sum_{n=1}^{\infty} \lambda_{n} \chi_{\left[0, t_{n}\right)}$, where

$$
\lambda_{n}=\frac{1}{n^{p+1 / p}\left(W\left(t_{n}\right)\right)^{1 / p}} .
$$

Clearly $f^{*}=f$. Consequently, in the case where $p \leq 1$, we have

$$
\begin{aligned}
\|f\|_{\Lambda^{p}(w)}^{p} & =\int_{0}^{\infty}\left(\sum_{n=1}^{\infty} \lambda_{n} \chi_{\left[0, t_{n}\right)}(t)\right)^{p} w(t) d t \\
& \leq \int_{0}^{\infty}\left(\sum_{n=1}^{\infty} \lambda_{n}^{p} \chi_{\left[0, t_{n}\right)}(t)\right) w(t) d t \\
& =\sum_{n=1}^{\infty} \lambda_{n}^{p} W\left(t_{n}\right)=\sum_{n=1}^{\infty} \frac{1}{n^{p^{2}+1}}<\infty .
\end{aligned}
$$

On the other hand, if $p>1$ we have, by the Minkowski inequality,

$$
\begin{aligned}
\|f\|_{\Lambda^{p}(w)} & =\left(\int_{0}^{\infty}\left(\sum_{n=1}^{\infty} \lambda_{n} \chi_{\left[0, t_{n}\right)}(t)\right)^{p} w(t) d t\right)^{1 / p} \\
& \leq \sum_{n=1}^{\infty}\left(\int_{0}^{\infty}\left(\lambda_{n} \chi_{\left[0, t_{n}\right)}(t)\right)^{p} w(t) d t\right)^{1 / p} \\
& =\sum_{n=1}^{\infty} \lambda_{n}\left(W\left(t_{n}\right)\right)^{1 / p}=\sum_{n=1}^{\infty} \frac{1}{n^{p+1 / p}}<\infty .
\end{aligned}
$$

Thus, in both cases, $f \in \Lambda^{p}(w)$. The function $g$ defined by $g(x)=f(-x)$ satisfies $g^{*}=f^{*}$, and so it too is in $\Lambda^{p}(w)$.

Now consider the function $h=f+g$. Its distribution function is twice the distribution function of $f$, and so $h^{*}(t)=f^{*}(t / 2)=\sum_{n=1}^{\infty} \lambda_{n} \chi_{\left[0,2 t_{n}\right)}(t)$. So, for 
each $m \in \mathbb{N}$, we have that

$$
\begin{aligned}
\|h\|_{\Lambda^{p}(w)}^{p} & =\int_{0}^{\infty}\left(\sum_{n=1}^{\infty} \lambda_{n} \chi_{\left[0,2 t_{n}\right)}(t)\right)^{p} w(t) d t \geq \int_{0}^{\infty} \lambda_{m}^{p} \chi_{\left[0,2 t_{m}\right)}(t) w(t) d t \\
& =\lambda_{m}^{p} W\left(2 t_{m}\right)=\frac{1}{m^{p^{2}+1}} \frac{W\left(2 t_{m}\right)}{W\left(t_{m}\right)}>\frac{2^{m}}{m^{p^{2}+1}} .
\end{aligned}
$$

Taking the limit as $m$ tends to $\infty$, we see that $h \notin \Lambda^{p}(w)$. This shows that $\Lambda^{p}(w)$ is not a linear space, and completes the proof that (iii) implies (i).

It remains to show that (i) implies (ii). We will do this indirectly, i.e., by showing that if (ii) does not hold then (i) does not hold. In the course of doing this we will also prove some of the implications of the corollary.

Suppose then that (ii) does not hold. Consider the sets $E_{n}$ defined by $E_{n}=\{t>$ $\left.0: W(2 t)>2^{n} W(t)\right\}$. They of course satisfy $E_{n+1} \subset E_{n}$. The fact that (ii) does not hold means that there exist a pair of positive numbers $\alpha$ and $\beta$ with $\alpha<\beta$ and some integer $N=N(\alpha, \beta)$ such that $E_{n} \subset(\alpha, \beta)$ for all $n \geq N(\alpha, \beta)$. This establishes that condition (ii) $)^{\prime}$ of the corollary holds for any choice of $\alpha$ and $\beta$ as above, provided we choose $C=2^{N(\alpha, \beta)}$.

Thus it is clear that, if we can show that conditions (i) ${ }^{\prime}$, (ii) ${ }^{\prime}$ and $(\text { iii) })^{\prime}$ of the corollary satisfy the two implications (ii) ${ }^{\prime} \Longrightarrow(\text { iii })^{\prime}$ and (iii) ${ }^{\prime} \Longrightarrow(\text { i })^{\prime}$, then this will certainly complete the proof that (i) implies (ii) and so will complete the proof of the theorem. At the same time we will have completed a considerable part of the proof of the corollary.

Accordingly we shall now prove that (ii) $)^{\prime} \Longrightarrow(\text { iii) })^{\prime}$.

Let $\alpha, \beta$ and $C$ be positive constants for which (ii) ${ }^{\prime}$ holds. If $\beta<\alpha$, then $W(2 t) \leq C W(t)$ for all $t>0$, i.e., $W$ satisfies condition (iii-A) ${ }^{\prime}$ with $C^{\prime}=C$. Thus we can suppose that $\alpha \leq \beta$. Let us first consider the case where $W(t)>$ 0 for all $t>0$. Then $W(2 t) / W(t)$ is a continuous function for all $t>0$ and is therefore bounded by some constant $C_{1}$ on the interval $[\alpha, \beta]$. It follows that $W(2 t) \leq \max \left\{C, C_{1}\right\} W(t)$ for all $t>0$, so again we have obtained condition (iii-A) $)^{\prime}$, this time with $C^{\prime}=\max \left\{C, C_{1}\right\}$.

It remains to consider the case where $W(t)=0$ for some positive $t$. In this case there exists $t_{0}>0$ such that $W(t)=0$ if and only if $t \in\left(0, t_{0}\right]$. Thus we obtain condition (iii-B) ${ }^{\prime}$ for $a=t_{0}, b=\beta$ and $C^{\prime}=C$.

Our final step will be to show that (iii) ${ }^{\prime} \Longrightarrow(\mathrm{i})^{\prime}$, i.e., that either of the conditions $(\text { iii-A })^{\prime}$ or (iii-B) $)^{\prime}$ is sufficient to imply that $\Lambda^{p}(w)$ is a linear space. In the case of condition (iii-A) ${ }^{\prime}$, which is exactly the $\Delta_{2}$-condition used in [11. Th. 1.1] (see also [8], [13), we can apply [11. Th. 1.1] (see also [8, Corollary 2.2, p. 482], [13 p. 6]) to obtain that $\|\cdot\|_{\Lambda^{p}(w)}$ is a quasinorm, which in turn immediately implies that $\Lambda^{p}(w)$ is a linear space.

If condition (iii-B)' holds, then we need a somewhat longer argument.

We define an auxiliary weight function $v:(0, \infty) \rightarrow[0, \infty)$ by $v(t)=1 / a$ for $t \in(0, a]$ and $v(t)=w(t)$ for $t \in(a, \infty)$. Let $V(t)=\int_{0}^{t} v(x) d x$. We claim that $V$ satisfies the $\Delta_{2}$-condition. To prove this claim we first note that $V(t)>0$ for all positive $t$. Furthermore, for all $t \in(0, a / 2]$ we have $V(2 t) / V(t)=2$. So, by obvious continuity considerations, it will suffice to show that $V(2 t) / V(t)$ is bounded on the interval $[\gamma, \infty)$ for some $\gamma>0$. There are two cases to be considered. Suppose first 
that $W$ is bounded, i.e., $W(t) \leq M$ for all $t>0$. Then, for all $t \geq a$, we have

$$
\frac{V(2 t)}{V(t)}=\frac{1+W(2 t)}{1+W(t)} \leq \frac{1+M}{1} \text {. }
$$

Alternatively, if $\lim _{t \rightarrow \infty} W(t)=\infty$, we can choose $\gamma$ sufficiently large so that $W(\gamma) \geq 1$ and $\gamma \geq b$. Then, for all $t \geq \gamma$, we have

$$
\frac{V(2 t)}{V(t)}=\frac{1+W(2 t)}{1+W(t)} \leq \frac{W(\gamma)+W(2 t)}{W(t)} \leq \frac{2 W(2 t)}{W(t)} \leq 2 C^{\prime}
$$

This proves our claim. Consequently it also shows, again by [11, Th. 1.1] (see also [8, Corollary 2.2], [13, p. 6]) that $\Lambda^{p}(v)$ is a linear space. We are now ready to use Lemma 1.2 to deduce that $\Lambda^{p}(w)$ is also a linear space:

Let $f$ and $g$ be arbitrary functions in $\Lambda^{p}(w)$. We need to show that $f+g \in \Lambda^{p}(w)$. Clearly $|f|$ and $|g|$ are also in $\Lambda^{p}(w)$, and it will suffice to show that $|f|+|g| \in \Lambda^{p}(w)$, i.e. we can assume that $f$ and $g$ are non-negative functions. By Lemma 1.2 the functions $\min \{f, 1\}$ and $\min \{g, 1\}$ are both in $\Lambda^{p}(v)$. Furthermore, it is easy to check that

$$
\min \{f+g, 1\} \leq 2(\min \{f, 1\}+\min \{g, 1\}) .
$$

(Obviously (2.1) holds at the points where $f+g \leq 1$, and if $f+g>1$ then at least one of $f$ and $g$ must be greater than $1 / 2$, which again ensures that (2.11) holds.) We deduce that $\min \{f+g, 1\} \in \Lambda^{p}(v)$, and another application of Lemma 1.2 shows that $f+g \in \Lambda^{p}(w)$.

This completes the proof of the theorem. But we still need to obtain one last implication in the corollary, namely that (i $)^{\prime} \Longrightarrow(\mathrm{ii})^{\prime}$.

Again we shall use an indirect approach, showing that if (ii) ${ }^{\prime}$ does not hold, then neither does (i)':

Indeed, if (ii)' does not hold, then, for each positive $\alpha, \beta$ and $C$, there must exist $t=t(\alpha, \beta, C) \in(0, \alpha] \cup[\beta, \infty)$ such that $W(2 t)>C W(t)$. In particular, the sequence $\left\{t\left(1 / m, m, 2^{m}\right)\right\}_{m \in \mathbb{N}}$, must have a subsequence with the properties stated in condition (ii) of the theorem. We have already seen that this implies (iii), which in turn implies (i), i.e., that $\Lambda^{p}(w)$ is not a linear space, exactly as is required to complete our proof.

Proof of Theorem [1.6. Clearly, the parameter $p$ is immaterial here, so we can with no loss of generality assume that $p=1$. Suppose that (ii) is satisfied. Then, as shown in the proof of Theorem 1.4 there exists a monotone (either increasing or decreasing) sequence $\left\{t_{n}\right\}$ such that $W\left(t_{n}\right)>0$ and

$$
\frac{W\left(2 t_{n}\right)}{W\left(t_{n}\right)} \rightarrow \infty, \quad n \rightarrow \infty
$$

We set

$$
f(t)=\sum_{n=1}^{\infty} \frac{1}{W\left(t_{n}\right)} \chi_{\left[s_{n}, t_{n}\right)},
$$

where

$$
s_{n}= \begin{cases}t_{n-1} & \text { when }\left\{t_{n}\right\} \text { is increasing; } \\ t_{n+1} & \text { when }\left\{t_{n}\right\} \text { is decreasing }\end{cases}
$$


and $t_{0}=0$. In both cases,

$$
\begin{aligned}
\|f\|_{\Lambda^{1, \infty}(w)} & =\sup _{0<t<\infty} f^{*}(t) W(t)=\sup _{n \in \mathbb{N}} \sup _{s_{n}<t<t_{n}} f^{*}(t) W(t) \\
& =\sup _{n \in \mathbb{N}} f^{*}\left(s_{n}\right) W\left(t_{n}\right)=1,
\end{aligned}
$$

while, for $h$ as in the proof of Theorem 1.4 ,

$$
\begin{aligned}
\|h\|_{\Lambda^{1, \infty}(w)} & =\sup _{0<t<\infty} f^{*}\left(\frac{t}{2}\right) W(t)=\sup _{0<t<\infty} f^{*}(t) W(2 t) \\
& =\sup _{n \in \mathbb{N}} \sup _{s_{n}<t<t_{n}} f^{*}(t) W(2 t)=\sup _{n \in \mathbb{N}} f^{*}\left(s_{n}\right) W\left(2 t_{n}\right)=\frac{W\left(2 t_{n}\right)}{W\left(t_{n}\right)} \rightarrow \infty .
\end{aligned}
$$

The proof of the converse implication is analogous to that in Theorem 1.4

\section{EXAMPLES}

Example 3.1. We will find a measurable function $w:(0, \infty) \rightarrow[0, \infty)$ such that the two functions $W(x)=\int_{0}^{x} w(t) d t$ and $\Phi(x)=\int_{x}^{\infty} t^{-p} w(t) d t$ are both finite for all $x>0$ but the set $\Lambda^{p}(w)=\left\{f: \int_{0}^{\infty} f^{*}(t)^{p} w(t) d t<\infty\right\}$ is not a linear space.

These conditions on $W$ and $\Phi$ are apparently necessary and sufficient to ensure that the space $\Gamma^{p}(w)$ is non-trivial, so it seems relevant to impose them here.

Initially the $w$ which we construct can assume the value 0 . But, as we shall see, it is easy to modify this to an example where $w$ is strictly positive.

Here is the construction:

Let us first define a sequence of positive numbers $w_{n}$ recursively by setting $w_{1}=1$ and

$$
w_{n}=\left(2^{n}-1\right)\left(w_{1}+w_{2}+\ldots+w_{n-1}\right)
$$

for all $n>1$. Then we define a second sequence of positive numbers $s_{n}$ recursively by setting $s_{1}=2$ and, for each $n>1$,

$$
s_{n}=\max \left\{2 s_{n-1}, w_{n}^{1 / p} 2^{n / p}+1\right\} .
$$

The function $w:(0, \infty) \rightarrow[0, \infty)$ is defined by $w=\sum_{n=1}^{\infty} w_{n} \chi_{\left(s_{n}-1, s_{n}\right]}$. It follows from (3.2) that $w_{n} /\left(s_{n}-1\right)^{p} \leq 2^{-n}$, and so

$$
\Phi(0)=\int_{0}^{\infty} t^{-p} w(t) d t=\sum_{n=1}^{\infty} w_{n} \int_{s_{n}-1}^{s_{n}} t^{-p} d t \leq \sum_{n=1}^{\infty} w_{n}\left(s_{n}-1\right)^{-p} \leq 1 .
$$

Obviously we also have $\int_{0}^{x} w(t) d t<\infty$ for each $x>0$. Thus $w$ satisfies the conditions mentioned above which ensure that $\Gamma^{p}(w)$ is non-trivial.

We claim that for this particular choice of $w$, the set $\Lambda^{p}(w)$ is not a linear space.

To show this, let us first observe that, by (3.2), we have $s_{n-1} \leq s_{n} / 2 \leq s_{n}-1$, and so

$$
\int_{0}^{s_{n-1}} w(t) d t=\int_{0}^{s_{n} / 2} w(t) d t=w_{1}+w_{2}+\ldots+w_{n-1} .
$$

It follows, using (3.1), that

$$
\begin{aligned}
W\left(s_{n}\right) & =\int_{0}^{s_{n}} w(t) d t=w_{1}+w_{2}+\ldots+w_{n-1}+w_{n} \\
& =2^{n}\left(w_{1}+w_{2}+\ldots+w_{n-1}\right)=2^{n} \int_{0}^{s_{n} / 2} w(t) d t=2^{n} W\left(s_{n} / 2\right) .
\end{aligned}
$$


Thus the sequence $\left\{t_{n}\right\}$ given by $t_{n}=s_{n} / 2$ tends to $\infty$ and satisfies

$$
W\left(2 t_{n}\right)=2^{n} W\left(t_{n}\right) \text {. }
$$

In other words, $w$ satisfies condition (iii) of Theorem 1.4. Consequently $\Lambda^{p}(w)$ is not a linear space.

To get another less exotic example, i.e., where the weight function is strictly positive, we simply replace $w$ by $w+u$, where $u$ is any strictly positive function for which the above function $f$ satisfies

$$
\int_{0}^{\infty} f(t)^{p} u(t) d t<\infty \text { and } \int_{t}^{\infty} s^{-p}(w(s)+u(s)) d s<\infty .
$$

Example 3.2. Let $w=\chi_{[1, \infty)}$. Then $\Lambda^{p}(w)$ is not quasinormable but is a linear space.

\section{ORLICZ-LORENTZ SPACES}

To conclude this note we present a result for Orlicz-Lorentz spaces which is similar to Corollary 1.5. Let $\varphi:[0, \infty) \rightarrow[0, \infty)$ be an Orlicz function, that is, $\varphi(0)=0, \varphi$ is strictly increasing and $\lim _{u \rightarrow \infty} \varphi(u)=\infty$. As in Definition 1.1, let $w:(0, \infty) \rightarrow[0, \infty)$ be a weight such that $W$ is finite everywhere. By $\Lambda_{\varphi, w}$ we denote the Orlicz-Lorentz space [12] defined as follows:

$$
\Lambda_{\varphi, w}=\left\{f \text { measurable }: I_{\varphi}(\lambda f):=\int_{0}^{\infty} \varphi\left(\lambda f^{*}(t)\right) w(t) d t<\infty \text { for some } \lambda>0\right\} .
$$

Observe that if $\varphi(u)=u^{p}$, then $\Lambda_{\varphi, w}=\Lambda^{p}(w)$, and if the weight function $w$ is constant, then $\Lambda_{\varphi, w}$ becomes an Orlicz space ([4, [19]). (Note that if $W$ were permitted to assume infinite values then $\Lambda_{\varphi, w}$ would either be trivial, or not a linear space, by essentially the same arguments as given in Remark 1.3 for $\Lambda^{p}(w)$.) We will see that the $\Delta_{2}$-condition (cf. (1.4) ) plays an important role here, not only for the function $W$, but also for the function $\varphi$.

Theorem 4.1. Assume that $\varphi$ satisfies the $\Delta_{2}$-condition and that $w$ is strictly positive. Then $\Lambda_{\varphi, w}$ is a linear space if and only if $W$ satisfies the $\Delta_{2}$-condition.

Proof. Since $w(t)>0$, we also have $W(t)>0$ for all $t>0$. If $W$ does not satisfy the $\Delta_{2}$-condition, then there exists a sequence $\left\{t_{n}\right\}_{n=1}^{\infty} \subset(0, \infty)$ such that $W\left(2 t_{n}\right)>2{ }^{n} W\left(t_{n}\right)$. We will show that this implies that $\Lambda_{\varphi, w}$ is not linear. In view of the assumption that $W$ is finite and thus continuous, by passing, if necessary, to a subsequence we can assume that $\left\{t_{n}\right\}$ converges either to zero or infinity. We shall only consider the case where $t_{n} \rightarrow \infty$. The other case can be treated analogously. Without loss of generality we can also assume that $2 t_{n}<t_{n+1}$, for all $n \in \mathbf{N}$, and also for $n=0$, after we define $t_{0}=0$. For

$$
\lambda_{n}=\varphi^{-1}\left(\frac{1}{n^{2} W\left(t_{n}\right)}\right)
$$

define

$$
f(t)=\sum_{n=1}^{\infty} \lambda_{n} \chi_{\left[t_{n-1}, t_{n}\right)}(t)
$$

Obviously $f^{*}=f$ and

$$
I_{\varphi}(f) \leq \sum_{n=1}^{\infty} \varphi\left(\lambda_{n}\right) W\left(t_{n}\right)=\sum_{n=1}^{\infty} n^{-2}<\infty .
$$


Defining $g(t)=f(-t)$ and $h=f+g$, we have $h^{*}(t)=f^{*}(t / 2)$. Thus, in view of the $\Delta_{2}$-condition on $\varphi$, for any $\lambda>0$ there exists $k(\lambda)>0$ such that $\varphi(\lambda u) \geq k(\lambda) \varphi(u)$ for all $u \geq 0$, and so

$$
\begin{aligned}
I_{\varphi}(\lambda h) & =\sum_{n=1}^{\infty} \varphi\left(\lambda \lambda_{n}\right)\left[W\left(2 t_{n}\right)-W\left(2 t_{n-1}\right)\right] \\
& \geq k(\lambda) \sum_{n=1}^{\infty} \varphi\left(\lambda_{n}\right)\left[2^{n} W\left(t_{n}\right)-W\left(t_{n}\right)\right] \\
& =k(\lambda) \sum_{n=1}^{\infty} \frac{2^{n}-1}{n^{2}}=\infty,
\end{aligned}
$$

which shows that $h \notin \Lambda_{\varphi, w}$.

Now, conversely, assuming that $W$ satisfies the $\Delta_{2}$-condition, we will show that $\Lambda_{\varphi, w}$ is linear. We first observe that the $\Delta_{2}$-condition on $W$ implies that

$$
\int_{0}^{\infty} u\left(\frac{t}{2}\right) w(t) d t \leq C \int_{0}^{\infty} u(t) w(t) d t
$$

for every non-increasing function $u$. (This is obvious when $u$ is of the form $u=$ $a \chi_{[0, b)}$, where $a$ and $b$ are positive constants. So it also holds for all finite sums of such functions and for all monotone limits of such sums, i.e., for all non-increasing functions.) If $f, g \in \Lambda_{\varphi, w}$, then $I_{\varphi}(\lambda f)<\infty$ and $I_{\varphi}(\lambda g)<\infty$ for some $\lambda>0$, and, using (4.1), we have

$$
\begin{aligned}
I_{\varphi}\left(\frac{\lambda}{2}(f+g)\right) & =\int_{0}^{\infty} \varphi\left(\left(\frac{\lambda}{2} f+\frac{\lambda}{2} g\right)^{*}(t)\right) w(t) d t \\
& \leq \int_{0}^{\infty} \varphi\left(\frac{\lambda}{2} f^{*}\left(\frac{t}{2}\right)+\frac{\lambda}{2} g^{*}\left(\frac{t}{2}\right)\right) w(t) d t \\
& \leq C \int_{0}^{\infty} \varphi\left(\frac{\lambda}{2} f^{*}(t)+\frac{\lambda}{2} g^{*}(t)\right) w(t) d t \\
& \leq C \int_{0}^{\infty} \varphi\left(\lambda \max \left(f^{*}(t), g^{*}(t)\right) w(t) d t\right. \\
& \leq C\left(I_{\varphi}(\lambda f)+I_{\varphi}(\lambda g)\right)<\infty,
\end{aligned}
$$

which shows that $f+g \in \Lambda_{\varphi, w}$.

Remark 4.2. Theorem 4.1 can be considered as a generalization of Corollary 1.5 at least for strictly positive weights for which condition (iii-B)' has to be excluded.

\section{ACKNOWLEDGEMENT}

We thank the referee for a helpful comment.

\section{REFERENCES}

[1] M. Ariño and B. Muckenhoupt, Maximal functions on classical Lorentz spaces and Hardy's inequality with weights for nonincreasing functions, Trans. Amer. Math. Soc. 320 (1990), 727-735. MR 90k:42034

[2] J. Bastero, M. Milman and F. Ruiz Blasco, A note on $L(\infty, q)$ spaces and Sobolev embeddings, Indiana U. Math. J. 52 (2003), 1215-1230.

[3] C. Bennett, R. DeVore and R. Sharpley, Weak- $L^{\infty}$ and BMO, Annals of Math. 113 (1981), 601-611. MR 82h:46047 
[4] C. Bennett and R. Sharpley, Interpolation of Operators, Pure and Applied Mathematics Vol. 129, Academic Press, Boston, 1988. MR 89e:46001

[5] A. P. Calderón, Intermediate spaces and interpolation, the complex method, Studia Math. 24 (1964), 113-190. MR 29:5097]

[6] M. J. Carro, A. García del Amo and J. Soria, Weak-type weights and normable Lorentz spaces, Proc. Amer. Math. Soc. 124 (1996), 849-857. MR 96f:42022

[7] M. Carro, L. Pick, J. Soria and V. D. Stepanov, On embeddings between classical Lorentz spaces, Math. Ineq. Appl. 4 (2001), 397-428. MR 2002d:46026

[8] M. J. Carro and J. Soria, Weighted Lorentz spaces and the Hardy operator, J. Funct. Anal. 112 (1993), 480-494. MR 94f:42025

[9] M. J. Carro and J. Soria, Boundedness of some integral operators, Canad. J. Math. 45 (1993), 1155-1166. MR 95d:47064

[10] M. J. Carro and J. Soria, The Hardy-Littlewood maximal function and weighted Lorentz spaces, J. London Math. Soc. 55 (1997), 146-158. MR 98g:42028

[11] A. Haaker, On the conjugate space of Lorentz space, Technical Report, Lund 1970, 1-23.

[12] A. Kamińska, Some remarks on Orlicz-Lorentz spaces, Math. Nachr. 147 (1990), 29-38. MR 92h:46034

[13] A. Kamińska and L. Maligranda, Order convexity and concavity in Lorentz spaces $\Lambda_{p, w}, 0<$ $p<\infty$, Studia Math. 160 (2004), 267-286.

[14] A. Kamińska and L. Maligranda, On Lorentz spaces $\Gamma_{p, w}$, Israel J. Math. 140 (2004), 285332.

[15] S. G. Krein, Ju. I. Petunin and E. M. Semenov, Interpolation of Linear Operators, Translations of Math. Monog., Providence 1982. MR 84j:46103

[16] G. G. Lorentz, On the theory of spaces $\Lambda$, Pacific J. Math. 1 (1951), 411-429. MR 13:470c

[17] J. Malý and L. Pick, An elementary proof of sharp Sobolev embeddings, Proc. Amer. Math. Soc. 130 (2001), 555-563. MR 2002j:46042

[18] J. Mari, A counterexample in power signals space, IEEE Trans. Automat. Control 41 (1996), 115-116. MR 96i:93004

[19] S. Mazur and W. Orlicz, On some classes of linear spaces, Studia Math. 17 (1958), 97-119. MR 20:4780

[20] J. Musielak and W. Orlicz, On generalized variations I, Studia Math. 18 (1959), 11-41. MR 21:3524

[21] P. B. Pierce and D. Waterman, $A \Delta_{2}$-equivalent condition, Real Anal. Exchange 26 (2000/2001), 651-655. MR 2002e:26010

[22] E. Sawyer, Boundedness of classical operators on classical Lorentz spaces, Studia Math. 96 (1990), 145-158. MR 91d:26026

[23] N. Wiener, Generalized harmonic analysis, Acta Math. 55 (1930), 117-258.

Department of Mathematics, Technion-Israel Institute of Technology, Haifa 32000, ISRAEL

E-mail address: mcwikel@math.technion.ac.il

Department of Mathematical Sciences, The University of Memphis, Memphis, TenNESSEE 38152

E-mail address: kaminska@memphis.edu

Department of Mathematics, Lule̊̊ University of Technology, SE-971 87 Lule̊̊, SweDEN

E-mail address: lech@sm.luth.se

Department of Mathematical Analysis, Faculty of Mathematics and Physics, Charles University, Sokolovská 83, 18675 Praha 8, Czech Republic - and - Department of Mathematics, Brock University, 500 Glenridge Ave., St. Catharines, Ontario, Canada L2S 3A1

E-mail address: pick@karlin.mff.cuni.cz 